\title{
Electric arc furnace power quality analysis based on a stochastic arc model
}

\author{
Dariusz Grabowski, Janusz Walczak, Maciej Klimas \\ Faculty of Electrical Engineering \\ Silesian University of Technology \\ Gliwice, Poland \\ dariusz.grabowski@polsl.pl
}

\begin{abstract}
Electric arc furnaces (EAF) are commonly used in steel industry. Unfortunately, nonlinear characteristic of the arc furnace and its stochastic behavior bring about many problems, e.g. voltage flicker and waveform distortions. The AC furnaces are usually modelled using combined models which divide the phenomenon taking place in real objects into a deterministic and a stochastic or chaotic parts. The former can be expressed by a nonlinear differential equations. The paper goal was to take advantage of the earlier results, i.e. a closed form of the solution to one of the most popular nonlinear differential equations used for the AC electric arc modelling, and start research towards a complete EAC model which covers not only the deterministic but also the time-varying nature of the arc waveforms. Such a model can be helpful, among others, in solution to power quality problems in EAF supplying networks. Theoretical considerations have been illustrated by some examples and the results have been compared with measurements.
\end{abstract}

Keywords-electric arc furnace; nonlinear differential equations; power quality; stochastic model;

\section{INTRODUCTION}

Electric arc furnaces (EAFs) are used for melting metals in steel industry. A reliable EAF model is especially required during the design stage of new steel plants in order to estimate the possible problems with power quality [1], among others, waveform distortions and voltage flicker at the point of common coupling (PCC), and to undertake some actions to reduce the negative effects on the power network and loads in the adjacent area.

The development of a realistic arc model is a demanding task because of its stochastic and nonlinear nature [1], [2]. The arc ignites and extinguishes in a random way especially in the beginning of the EAF work cycle. The arc voltage-current (V-I) characteristic changes during the melting process. Most of the models worked out so far are two-step - they consist of a deterministic part extended further with the help of chaotic, stochastic or modulated components [3], [4], [5], [6], [7]. Modelling of the deterministic V-I characteristic can be made using, among others, nonlinear differential equations [5], [6], [8], [9], piece-wise linear approximations [4], mixed approximations using exponential and linear functions [4], approximations using shifted and amplified step functions [10], and the frequency domain approach is also possible [11]. Those approaches have different complexity and modelling accuracy.
In this paper a stochastic approach to EAF modelling based on a nonlinear differential equation has been proposed and preliminarily verified using real EAF data. The proposed solution is data driven - the model can be adapted to any given current waveform. Moreover, the proposed model is rather aimed at reproducing waveforms having the same statistics as measured ones than at exactly predicting the output waveforms for a given input signal.

\section{ANALYSIS OF EAF CURRENT AND VOLTAGE SignAlS}

The extraordinary arc phenomena and EAF analysis attracts researchers all over the world. The reactive power and waveform distortions introduced by EAFs into the network make their modelling very important not only form the theoretical but also from the practical point of view [1], [2], [3], [4], [5], [6], [12].

A simplified diagram of a typical supplying system of the EAF has been presented in Fig. 1. It includes a supplying $\mathrm{HV} / \mathrm{MV}$ transformer denoted by $\mathrm{T}_{1}$ and an electric arc furnace $\mathrm{MV} / \mathrm{LV}$ transformer denoted by $\mathrm{T}_{2}$. Moreover, a parameter $X_{\mathrm{LSC}}$ represents the short circuit reactance at the Bus 1 which is the point of common coupling, parameters $L_{\mathrm{f}}$ and $R_{\mathrm{f}}$ represent inductance and resistance of the feeder between transformers $\mathrm{T}_{1}$ and $\mathrm{T}_{2}$, parameters $L \mathrm{c}$ and $R \mathrm{c}$ represent the equivalent impedance of the flexible cables, bus conductors and graphite electrodes.

It must be stressed that the EAF current distortion is relatively low, especially in comparison with the voltage. The average total harmonic distortion coefficient for current (THDI) and voltage (THDV) for typical waveforms captured during the melting stage of the EAF cycle are equal to $4.0 \%$ and $23.5 \%$, respectively. The average harmonic content for these waveforms has been presented in Table I.

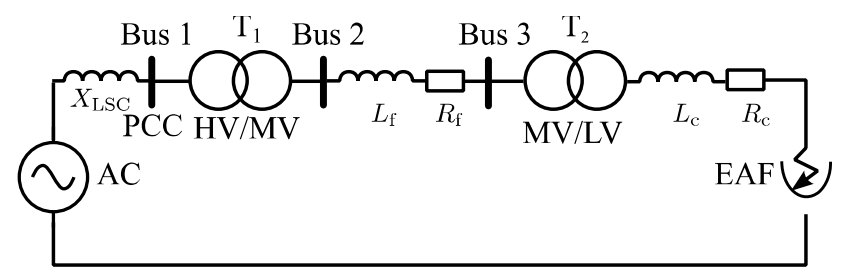

Fig. 1. Single line diagram of a typical electric arc furnace supplying system

(C) IEEE 2018 URL: http://ieeexplore.ieee.org/stamp/stamp.jsp?tp=\&arnumber=8494547\&isnumber=8493486

D. Grabowski, J. Walczak and M. Klimas, "Electric Arc Furnace Power Quality Analysis Based on a Stochastic Arc Model," 2018 IEEE International Conference on Environment and Electrical Engineering and 2018 IEEE Industrial and Commercial Power Systems Europe (EEEIC / I\&CPS Europe), Palermo, 2018, pp. 1-6.

DOI: 10.1109/EEEIC.2018.8494547 
TABLE I. EAF CURRENT AND VOLTAGE EXEMPLARY HARMONIC CONTENT For THE MELTing STAGE

\begin{tabular}{|c|c|c|}
\hline $\mathbf{h}$ & Current & Voltage \\
\hline 2 & $0.7 \%$ & $1.9 \%$ \\
\hline 3 & $3.2 \%$ & $21.4 \%$ \\
\hline 4 & $0.3 \%$ & $0.7 \%$ \\
\hline 5 & $2.1 \%$ & $8.1 \%$ \\
\hline 6 & $0.2 \%$ & $0.6 \%$ \\
\hline 7 & $1.0 \%$ & $3.7 \%$ \\
\hline 8 & $0.1 \%$ & $0.4 \%$ \\
\hline 9 & $0.3 \%$ & $2.2 \%$ \\
\hline 10 & $0.1 \%$ & $0.3 \%$ \\
\hline
\end{tabular}

The calculated THD coefficients as well as harmonic content, describing waveform distortions and presented in Table I, are consistent with results reported by other researchers [13], [14]. Exemplary arc current and arc voltage waveforms, acquired during the melting stage of the EAF work cycle, have been presented in Fig. 2.

Besides the distortion level, analysis of the real current and voltage waveforms acquired at the secondary windings of the EAF supplying transformer (Fig. 1) reveals a sort of amplitude modulation phenomenon (Fig. 3 and 4). It can be observed that the modulation parameters are changing randomly with time, so the current and voltage are, in fact, stochastic processes.

\section{EAF DETERMINISTIC MODEL}

The proposed stochastic approach to EAF modelling is based on a deterministic model expressed by a nonlinear differential equation that comes from the instantaneous power balance equation [5], [6], [8]. The other approaches consist in approximation based on a step function [10], [15], a piece-wise linear or an exponential approximation [4] and a neural network black-box model [16].

The application of the power balance law, which is the starting point of new EAF models, results in the following nonlinear differential equation describing a single-phase electric arc [8]:

$$
k_{1} r^{n}(t)+k_{2} r(t) \frac{\mathrm{d} r(t)}{\mathrm{d} t}=\frac{k_{3}}{r^{m+2}(t)} i^{2}(t)
$$

where $r(t)$ - the arc radius, $i(t)$ - the arc current, $k_{1}, k_{2}, k_{3}$ - the model coefficients determined experimentally, $n$ and $m-$ the equation parameters.

In this equation the arc radius is regarded as an unknown variable and the arc current $i(t)$ as input data. Subsequently, the arc voltage $u(t)$ can be determined using the following expression [8]:

$$
u(t)=\frac{k_{3}}{r^{m+2}(t)} i(t)
$$

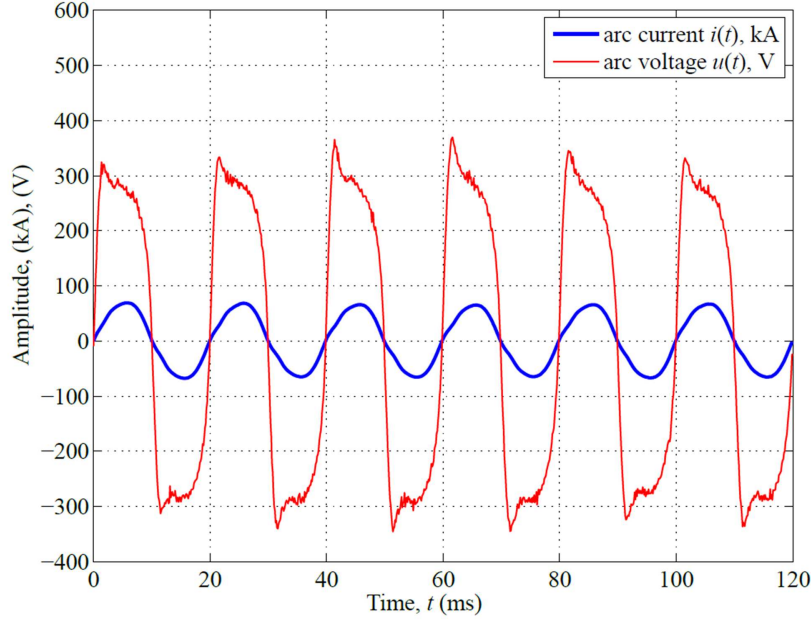

Fig. 2. Exemplary arc current and voltage waveforms

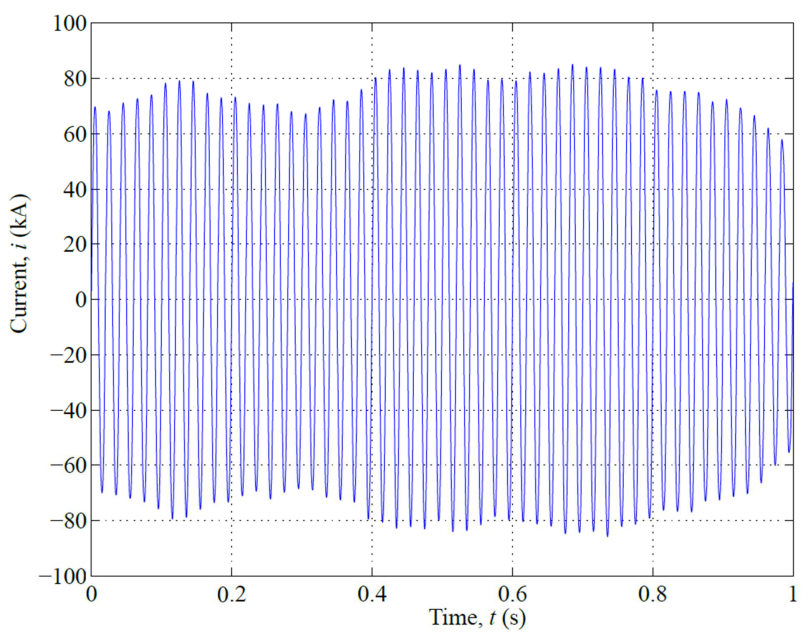

Fig. 3. Exemplary arc current waveform

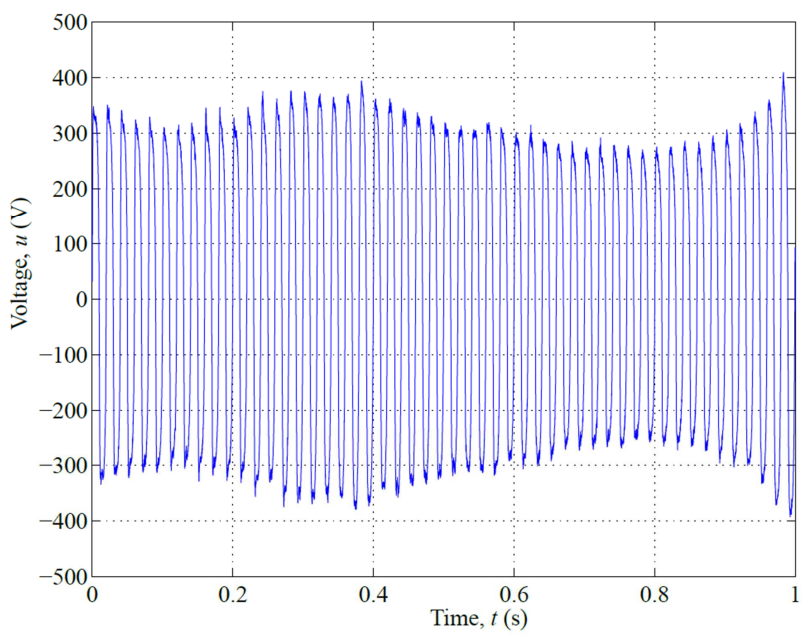

Fig. 4. Arc voltage waveform corresponding to the arc current in Fig. 3

(C) IEEE 2018 URL: http://ieeexplore.ieee.org/stamp/stamp.jsp?tp=\&arnumber=8494547\&isnumber=8493486

D. Grabowski, J. Walczak and M. Klimas, "Electric Arc Furnace Power Quality Analysis Based on a Stochastic Arc Model," 2018 IEEE International Conference on Environment and Electrical Engineering and 2018 IEEE Industrial and Commercial Power Systems Europe (EEEIC / I\&CPS Europe), Palermo, 2018, pp. 1-6.

DOI: 10.1109/EEEIC.2018.8494547 
The arc equation (1) for $n=2$ and $m=0,1$ or 2 can be brought to a linear equation [17], [18]. Its solution is expressed by [19]:

$$
y(t)=\beta \frac{k_{3}}{k_{1}} \mathrm{e}^{-\beta t} \int_{0}^{t} i^{2}(\tau) \mathrm{e}^{\beta \tau} \mathrm{d} \tau,
$$

where:

$$
\begin{aligned}
& y(t)=r^{m+4}(t), \\
& \beta=(m+4) \frac{k_{1}}{k_{2}},
\end{aligned}
$$

and finally the arc voltage can be determined using (2).

It can be proved that if the arc current is approximated by a sinusoidal waveform (see Section II):

$$
i(t)=\sqrt{2}|I| \sin (\omega t),
$$

then determination of auxiliary function (3) and subsequent application of (2) and (4) results in a closed form formula expressing the steady state arc voltage:

$$
u(t)=A(1-B \sin (2 \omega t+\psi))^{-l} \sin (\omega t),
$$

where:

$$
\begin{gathered}
A=\sqrt{2} k_{1}^{\frac{m+2}{m+4}} k_{3}^{\frac{2}{m+4}}|I|^{\frac{-m}{m+4}}, \\
B=\frac{1}{\sqrt{1+C^{2}}}, \\
C=\frac{2 \omega k_{2}}{(m+4) k_{1}}, \\
\psi=\arctan C^{-1}, \\
l=\frac{m+2}{m+4} .
\end{gathered}
$$

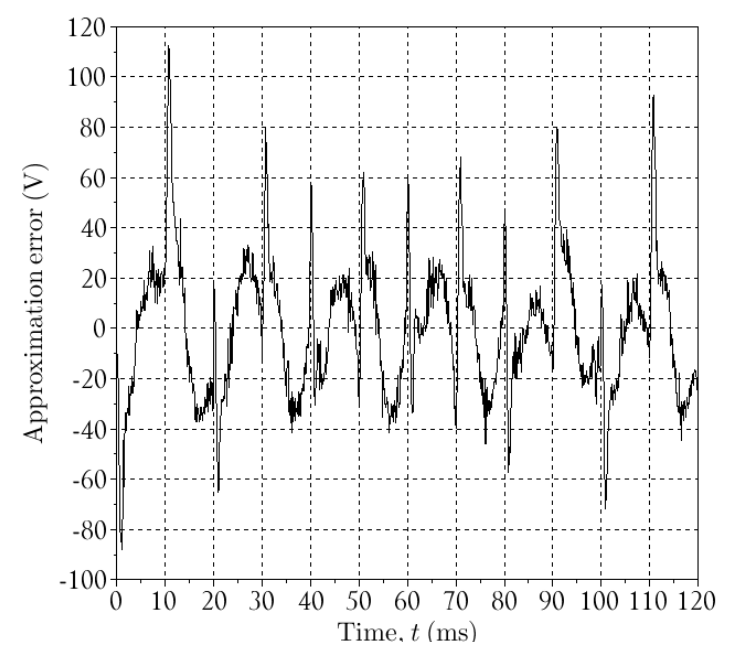

Fig. 5. Exemplary approximation error for the deterministic model
This is the starting point for building an EAF stochastic model. Its necessity is obvious if one compares measured waveforms and the deterministic model output signal defined by (7). An exemplary approximation error determined for the arc voltage waveform presented in Fig. 2 has been shown in Fig. 5. The error level does usually not exceed $10 \%$ except to times for which voltage crosses zero and error rises to $25 \%$.

\section{Stochastic ApproAch to EAF MODELling}

The input current signal of a stochastic model can be represented by a stochastic process $I(t)$. Obviously, the time domain description given by differential equation (1) and its solution given by (3) may be formally applied to calculate the system response to any single realization $i(t)$ of the input stochastic process $I(t)$. The set of these system responses can be then analyzed statistically. The whole set of input realizations, i.e. the input stochastic process $I(t)$, and the corresponding set of output realizations, i.e. the output stochastic process $U(t)$ representing the arc voltage, can be considered.

In this paper, we have focused on a short time analysis. The analysis of Fig. 2 and Fig. 3 leads to a conclusion that the EAF modelling can be carried out individually for consecutive time intervals in which the current is a sinusoidal deterministic waveform with constant amplitude and frequency. The lengths of these intervals can be as long as a few periods and depend on the EAF work cycle. Such an approach, which can be called a short time modelling, has been applied further.

It can be proved that if the realization of stochastic process $I(t)$ representing the arc current for a short time is sinusoidal then the corresponding realization of stochastic process $U(t)$ representing the arc voltage defined by (2) is expressed by (7) assuming its parameters and coefficients are constant.

Let us introduce stochastic behavior to the solution in the simplest possible way, i.e. by means of an additional random variable $\Gamma$ with a zero mean value and a given probability density function:

$$
U(t)=(1+\Gamma) A(1-B \sin (2 \omega t+\psi))^{-l} \sin (\omega t) .
$$

Some exemplary V-I characteristics obtained assuming that $n=2, m=0, k_{1}=2565, k_{2}=7.04, k_{3}=26.7$ [20], and random variable $\Gamma$ has uniform distribution $\mathcal{U}(-0.3,0.3)$ have been presented in Fig. 6. The arc voltage waveforms obtained in this case have been presented in Fig. 7.

Using the proposed approach, we can model random voltage amplitude changes but the shape of the waveform remains the same - see Fig. 7. In order to take into account the shape changes the stochastic model should be rather based on a stochastic process $\Gamma(t)$ than a random variable $\Gamma$ :

$$
U(t)=(1+\Gamma(t)) A(1-B \sin (2 \omega t+\psi))^{-l} \sin (\omega t) .
$$

Exemplary realizations of the process $\Gamma(t)$ calculated on the base of measurement data presented in Fig. 2 and the deterministic solution (7) have been presented in Fig. 8. The noisy spikes due to numerical errors, arising when the arc voltage crosses zero, have been removed by a low-pass filter.

(C) IEEE 2018 URL: http://ieeexplore.ieee.org/stamp/stamp.jsp?tp=\&arnumber=8494547\&isnumber=8493486

D. Grabowski, J. Walczak and M. Klimas, "Electric Arc Furnace Power Quality Analysis Based on a Stochastic Arc Model," 2018 IEEE International Conference on Environment and Electrical Engineering and 2018 IEEE Industrial and Commercial Power Systems Europe (EEEIC / I\&CPS Europe), Palermo, 2018, pp. 1-6. 


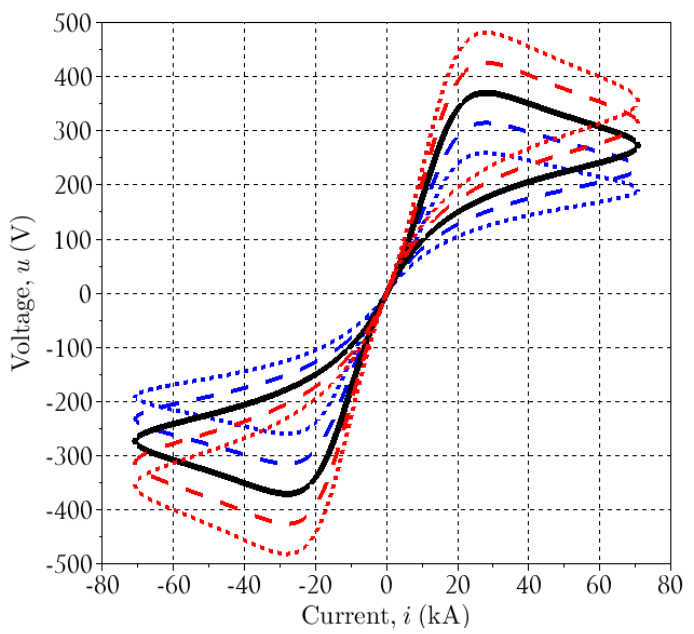

Fig. 6. Arc voltage-current characteristics obtained from the basic deterministic characteristic (black solid line, $\Gamma=0$ ) by changing the equation parameter $\Gamma$ (red color for $\Gamma>0$, blue color for $\Gamma<0$ )

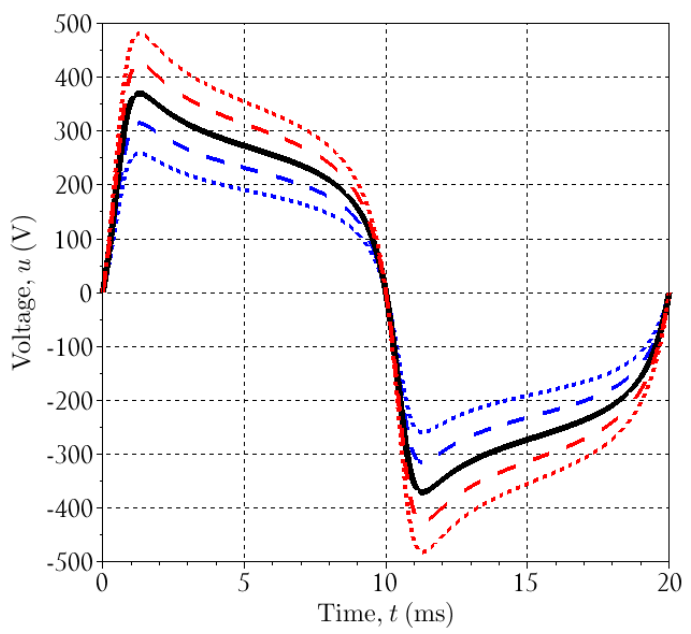

Fig. 7. Arc voltage waveforms corresponding to the characterics presented in Fig. 6 (black solid line, $\Gamma=0$; red color for $\Gamma>0$, blue color for $\Gamma<0$ )

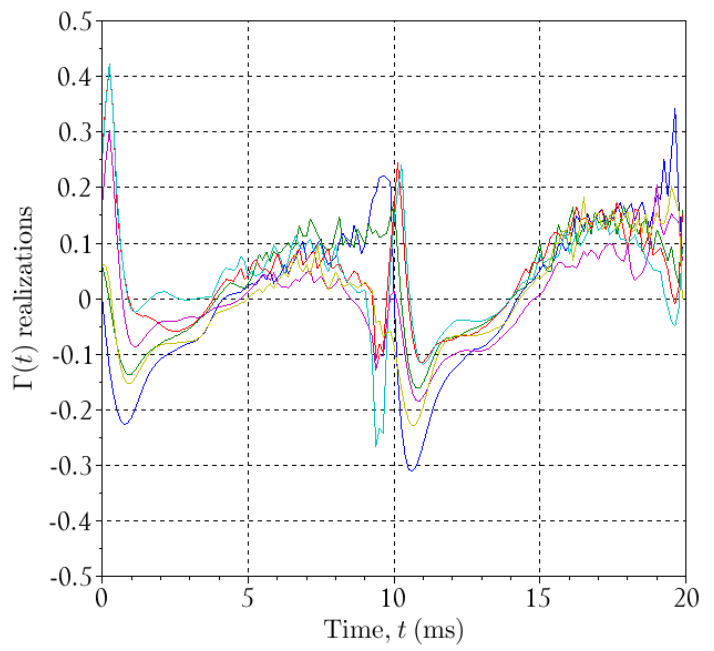

Fig. 8. Exemplary realization of the stochastic process $\Gamma(t)$
The identification of the stochastic process $\Gamma(t)$ will be performed in the future. On the base of measurement data some non-stationary stochastic processes or their superposition will be tested against the possible application in the model given by (14). Afterwards, having the description of the process $\Gamma(t)$, the arc voltage stochastic process can be easily described using stochastic moments [21]. Depending on the identification results, at least the first and the second order moments, i.e. mean value $\mu_{U}(t)$ and autocorrelation function $R_{U}\left(t_{1}, t_{2}\right)$, for the arc voltage stochastic process should be determined. These moments can be expressed with the help of the deterministic solution $u(t)$ given by (7) as well as the corresponding identified moments which describe the process $\Gamma(t)$, i.e. its mean value $\mu_{\Gamma}(t)$ and autocorrelation function $R_{\Gamma}\left(t_{1}, t_{2}\right)$ :

$$
\begin{gathered}
\mu_{U}(t)=\mathrm{E}[U(t)]=\left(1+\mu_{\Gamma}(t)\right) u(t), \\
R_{U}\left(t_{1}, t_{2}\right)=\left(1+\mu_{\Gamma}\left(t_{1}\right)+\mu_{\Gamma}\left(t_{2}\right)+R_{\Gamma}\left(t_{1}, t_{2}\right)\right) u\left(t_{1}\right) u\left(t_{2}\right),
\end{gathered}
$$

where $E[\cdot]$ denotes the expected value operator [21].

Stochastic modelling can be more specific and closer to the phenomena under consideration than general methodology expressed by (13) and (14). In this case, the following question must be answered: what makes the voltage waveforms stochastic if the input current is deterministic (see Fig. 2). For the model expressed by (1) there are two options:

- stochastic changes of the model coefficients $\left(k_{1}, k_{2}\right.$ and $k_{3}$ ),

- stochastic changes of the equation parameter $m$ assuming that analysis is carried out separately for each value of the parameter $n$.

The first option have been considered in [20] using Markov chains and Monte Carlo approach, so further we will focus on the second option assuming that $n=2$. It corresponds to the melting stage of the EAF work cycle - the most difficult one from the power quality point of view. Moreover, in this case the closed form deterministic solution expressed by (7) can be used as a starting point, too.

The voltage-current characteristics obtained assuming that parameter $n=2$ and parameter $m$ is a random variable $M$ with continuous uniform distribution $\mathcal{U}(-0.15,0.15)$ have been presented in Fig. 9. The uniform distribution has been chosen assuming that all $m$ belonging to a given interval are equally probable. The distribution support has been determined experimentally to reflect similar range of change of the V-I characteristics as for the approach based on (13) - see Fig. 6. The arc voltage waveforms obtained in this case have been presented in Fig. 10.

Experiments have proved that the parameter $m$ changes can cause similar results as observed for the simplest stochastic model expressed by (13). It has been illustrated in Fig. 11 by two exemplary characteristics obtained for the random variable $M$ taking value 0.1332 and for the random variable $\Gamma$ taking value -0.25 .

(C) IEEE 2018 URL: http://ieeexplore.ieee.org/stamp/stamp.jsp?tp=\&arnumber=8494547\&isnumber=8493486

D. Grabowski, J. Walczak and M. Klimas, "Electric Arc Furnace Power Quality Analysis Based on a Stochastic Arc Model," 2018 IEEE International Conference on Environment and Electrical Engineering and 2018 IEEE Industrial and Commercial Power Systems Europe (EEEIC / I\&CPS Europe), Palermo, 2018, pp. 1-6. 


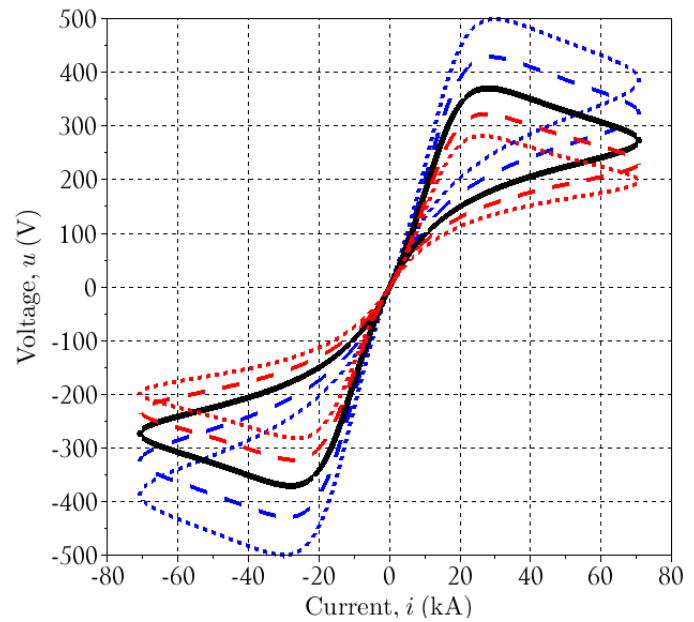

Fig. 9. Arc voltage-current characteristics obtained from the basic deterministic characteristic (black solid line, $m=0$ ) by changing the equation parameter $m$ (red color for $m>0$, blue color for $m<0$ )

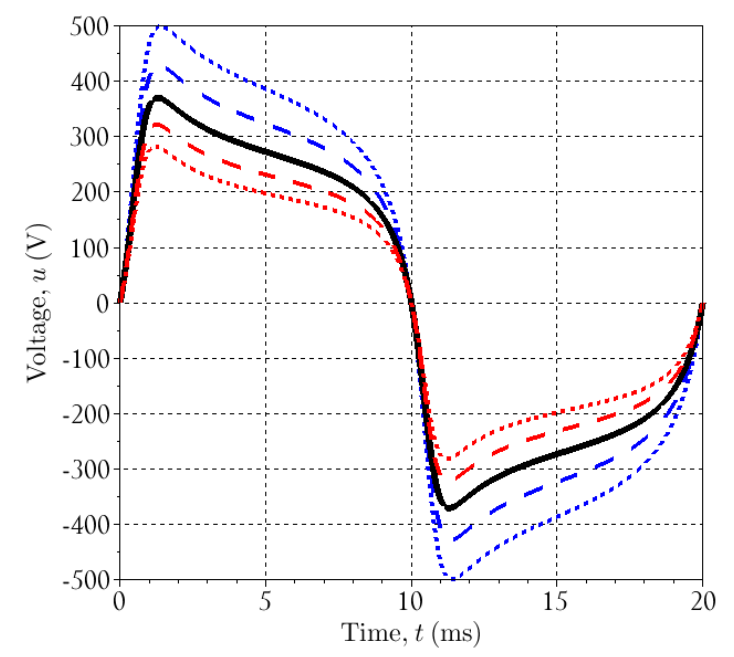

Fig. 10. Arc voltage waveforms corresponding to the characterics presented in Fig. 9 (black solid line, $m=0$; red color for $m>0$, blue color for $m<0$ )

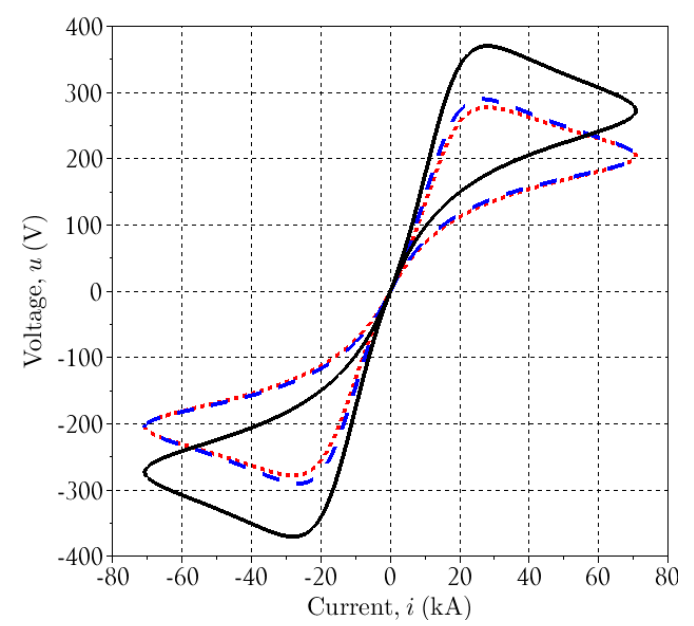

Fig. 11. Arc voltage-current characteristics obtained from the basic deterministic characteristic (black solid line) by changing $\Gamma$ (red solid line) and by changing the equation parameter $m$ (blue dashed line)

\section{CONCLUSIONS}

A stochastic EAF model can be obtained by transformation of a deterministic ODE expressing EAF power balance into a nonlinear random differential equation in which only the forcing term $I(t)$ and thus the solution $R(t)$ are stochastic processes. Such an approach is necessary if stochastic model describes EAF in longer time intervals. However, a short time analysis of measurement results have shown that in such a case the arc current can be approximated by a deterministic and sinusoidal waveform. So the EAF stochastic behavior can be the result of stochastic changes of either ODE parameters $(n$ and $m$ ) or power balance proportionality constants (model coefficients, i.e. $k_{1}, k_{2}$ and $k_{3}$ ). However, the simplest way of building the stochastic model consists in introducing a random variable into the deterministic model. It can be especially useful to model long time EAF behavior. The paper shows that different stochastic approaches can lead to similar stochastic EAF characteristics.

Future work includes taking into account both long time current and short time parameters/coefficients stochastic changes. The important task consists in the identification of the stochastic process $\Gamma(t)$ which will allow us to accurately reflect the real EAF behavior.

\section{ACKNOWLEDGMENT}

The authors would like to thank Prof. Haidar Samet (Shiraz University, Iran) for the EAF data.

\section{REFERENCES}

[1] J. Lavers and P. Biringer, "Real-time measurement of electric arcfurnace disturbances and parameter variations," IEEE Trans. on Industry Applications, vol. IA-22, no. 4, pp. 568-577, 1986.

[2] A. Sawicki, "Effective arc in AC-EAF modeling," Przegląd Elektrotechniczny, vol. 85, no. 12, pp. 263-269, 2009.

[3] M.F. Alvesa, Z.M.A. Peixotoa, C.P. Garciab, and D.G. Gomesc, "An integrated model for the study of flicker compensation in electrical networks," Electr. Power Syst. Research, vol. 80, no. 10, pp. 1299-1305, 2010 .

[4] M.A. Golkar and S. Meschi, "MATLAB modeling of arc furnace for flicker study," Proc. of the IEEE Int. Conf. on Industrial Technology, Chengdu, China, pp. 1-6, 2008.

[5] A.A. Gomez, J.J.M. Durango, and A.E. Mejia, "Electric arc furnace modeling for power quality analysis," Proc. of the IEEE ANDESCON Conf., Bogota, Colombia, pp. 1-6, 2010.

[6] O. Ozgun and A. Abur, "Development of an arc furnace model for power quality studies," Proc.of IEEE-PES Meeting, vol. 1, pp. 507-511, 1999.

[7] M. Esfahani and B. Vahidi, "A new stochastic model of electric arc furnace based on hidden Markov model: a study of its effects on the power system," IEEE Trans. on Power Delivery, vol. 27, no. 4, pp. 1893-1901, 2012.

[8] E. Acha, A. Semlyen, and N. Rajakovic, "A harmonic domain computational package for nonlinear problems and its applications to electric arcs," IEEE Trans. on Power Delivery, vol. 5, no. 3, pp. 1390 1397, 1990.

[9] A. Sawicki, "Problems of modeling an electrical arc with variable geometric dimensions," Przegląd Elektrotechniczny, vol. 89, no. 2b, pp. 270-275, 2013.

[10] M. Wciślik, "Characteristics of the symmetrical circuit of arc furnace in steady state during the melting," Przegląd Elektrotechniczny, vol. 87, no. 7 , pp. $68-71,2011$.

D. Grabowski, J. Walczak and M. Klimas, "Electric Arc Furnace Power Quality Analysis Based on a Stochastic Arc Model," 2018 IEEE International Conference on Environment and Electrical Engineering and 2018 IEEE Industrial and Commercial Power Systems Europe (EEEIC / I\&CPS Europe), Palermo, 2018, pp. 1-6. 
[11] P. Świszcz, "Frequency domain model of the electrical arc and its verification," Proc. of the 12th Int. Conf. on Environment and Electrical Engineering (EEEIC), Wrocław, Poland, pp. 164-168, 2013.

[12] H. Samet and A. Mojallal, "Enhancement of electric arc furnace reactive power compensation using Grey-Markov prediction method," IET Generation, Transmission \& Distribution, vol. 8, no. 9, pp. 1626-1636, 2014.

[13] H. Ghoudjehbaklou and A. Kargar, "Harmonic elimination of electric arc furnaces by active power filters and their stability analysis," COMPEL: The International Journal for Computation and Mathematics in Electrical and Electronic Engineering, vol. 21, no. 1, pp. 82-97, 2002.

[14] O. Salor, B. Gultekin, S. Buhan, B. Boyrazoglu, T. Inan, T. Atalik, A. Ak, A. Terciyanl, O. Unsar, E. Altntas, Y. Akkaya, E. Ozdemirci, I. Cadrc, and M. Ermis, "Electrical power quality of iron and steel industry in Turkey," IEEE Trans. on Industry Applications, vol. 46, no. 1, pp. 60-80, 2010.

[15] R.C. Bellido and T. Gomez, "Identification and modelling of a three phase arc furnace for voltage disturbance simulation," IEEE Trans. on Power Delivery, vol. 12, no. 4, pp. 1812-1817, 1997.
[16] G.W. Chang, Cheng-I Chen and Yu-Jen Liu, "A neural-network-based method of modeling electric arc furnace load for power engineering study," IEEE Trans. on Power Systems, vol. 25, no.1, pp. 138-146, 2010 .

[17] D. Grabowski and J. Walczak, "Analysis of deterministic model of electric arc furnace," Proc. of the 10th International Conference on Environment and Electrical Engineering EEEIC, Rome, Italy, pp. 1-4, 2011.

[18] D. Grabowski and J. Walczak, "Deterministic model of electric arc furnace - a closed form solution," COMPEL - The International Journal for Computation and Mathematics in Electrical and Electronic Engineering, vol. 32, no. 4, pp. 1428-1436, 2013.

[19] A.D. Polyanin and V.F. Zaitsev, Handbook of Exact Solutions for Ordinary Differential Equations. Boca Raton: CRC Press, 2003.

[20] D. Grabowski, Selected Applications of Stochastic Approach in Circuit Theory. Monograph. Gliwice: The Publishing House of Silesian University of Technology, 2015.

[21] A. Papoulis and S.Unnikrishna Pillai, Probability, Random Variables and Stochastic Processes. New York: McGraw Hill, 2002. 